\title{
$\phi$-FEM: a finite element method on domains defined by level-sets
}

\author{
Michel Duprez ${ }^{\dagger}$, Vanessa Lleras $^{\ddagger}$ and Alexei Lozinski* \\ † Équipe Inria MIMESIS, 1 place de l'Hôpital, 67000 Strasbourg, France \\ e-mail: michel.duprez@inria.fr \\ $\ddagger$ IMAG, Univ Montpellier, CNRS, Montpellier, France \\ e-mail: vanessa.lleras@umontpellier.fr \\ * Laboratoire de Mathématiques de Besançon, UMR CNRS 6623, \\ Université Bourgogne Franche-Comté, 16 route de Gray, 25030 Besançon, France. \\ e-mail: alexei.lozinski@univ-fcomte.fr
}

\begin{abstract}
We present a new finite element method, called $\phi$-FEM, to solve numerically elliptic partial differential equations using simple computational grids, not fitted to the boundary of the physical domain. The geometry of the domain is taken into account using a level-set function, which is a popular tool to deal with complicated or evolving domains. Our approach belongs to the family of fictitious domain methods (or immersed boundary methods) and is close to recent methods of cutFEM/XFEM type. Contrary to the latter, $\phi$-FEM does not need any non-standard numerical integration on cut mesh elements or on the actual boundary, while assuring the optimal convergence orders with finite elements of any degree and providing reasonably well conditioned discrete problems. The first version of $\phi$-FEM was introduced in [1] for the case of essential (Dirichlet) boundary conditions. The approximation to the solution is obtained there as a product of a finite element function with the given level-set function, also approximated by finite elements. The case of natural (Neumann or Robin) boundary conditions is less straightforward. A variant of $\phi$-FEM for this case is proposed in [2]. We introduce there the gradient of the primary solution as an auxiliary variable (only on the mesh cells cut by the boundary, so that the size of the numerical system is only slightly increased).

We shall outline the proofs of optimal convergence for our methods on the example of Poisson equation using Lagrange finite elements of any order. We shall also illustrate this by numerical tests and discuss the conditionning of resulting linear systems and the robustness with respect to the geometry. We shall conclude by presenting our ongoing work: an extension of our technique to the problems on evolving domains, such as simulations of a creeping flow (Stokes equations) around the moving particles.
\end{abstract}

\section{REFERENCES}

[1] M. Duprez and A. Lozinski. $\phi$-FEM: a finite element method on domains defined by level-sets. SIAM J. Numer. Anal. (2020), doi:10.1137/19M1248947

[2] M. Duprez, V. Lleras, and A. Lozinski, A new $\phi$-FEM approach for problems with natural boundary conditions. arXiv:2003.11733. 